(1)

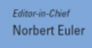
Journal of Nonlinear Mathematical Physics

\title{
Nonlinear Self-Adjointness and Conservation Laws for the Hyperbolic Geometric Flow Equation
}

Kênio A. A. Silva

To cite this article: Kênio A. A. Silva (2013) Nonlinear Self-Adjointness and Conservation Laws for the Hyperbolic Geometric Flow Equation, Journal of Nonlinear Mathematical Physics 20:1, 28-43, DOI: https://doi.org/10.1080/14029251.2013.792467

To link to this article: https://doi.org/10.1080/14029251.2013.792467

Published online: 04 January 2021 


\title{
Nonlinear Self-Adjointness and Conservation Laws for the Hyperbolic Geometric Flow Equation
}

\author{
Kênio A. A. Silva \\ Instituto de Matemática, Estatística e \\ Computação Científica \\ Universidade Estadual de Campinas \\ Rua Sérgio Buarque de Holanda, 651 \\ 13083-859 - Campinas - SP, Brasil. \\ kaasilva@ime.unicamp.br \\ Received 2 September 2012 \\ Accepted 12 October 2012
}

\begin{abstract}
We study the nonlinear self-adjointness of a class of quasilinear 2D second order evolution equations by applying the method of Ibragimov. Which enables one to establish the conservation laws for any differential equation. We first obtain conditions determining the self-adjointness for a sub-class in the general case. Then, we establish the conservation laws for hyperbolic geometric flow equation on Riemman surfaces.
\end{abstract}

Keywords: Nonlinear self-adjointness; conservation laws; hyperbolic geometric flow equation.

2000 Mathematics Subject Classification: 22E46, 53C35, 57S20

\section{Introduction}

Ibragimov recently introduced the concept of nonlinear self-adjointness and developed a technique for the determination of conservation laws for differential equations, [5-11]. Motivated by such work we propose to the study of self-adjointness and conservation laws for the following quasilinear 2D generalized second evolution equation

$$
u_{t t}=A u_{x y}+B u_{x} u_{y}+C u_{x x}+D u_{y y}+E u_{y}+F u_{x}+P u_{x}^{2}+Q u_{y}^{2}+G+H u_{t}+I u_{t}^{2},
$$

where the coefficients $A, B, C, D, E, F, P, Q, G, H, I$ are functions of the independent variables $t, x, y$ and the dependent variable $u=u(t, x, y)$. Among such equations we consider the hyperbolic geometric flow equation in isothermic coordinates $x, y$ on a Riemann surfaces, which has been introduced by Kong and Liu [12] to study the wave character of the Riemannian metric and curvature, the Ricci and the scalar curvatures, as singularities, existence and regularities of the flow solutions. In particular, this equation can be seen as the Einstein's hyperbolic geometric flow in vacuo. Here we consider an equation that generalizes the $2 \mathrm{D}$ hyperbolic flow equation. There are lot of nonlinear equations of type (1.1) arising in physics, chemistry and biology, [11].

In a recent paper we apply the method the Ibragimov to a class of evolution equations of type

$$
R u_{t}=A u_{x y}+B u_{x} u_{y}+C u_{x x}+D u_{y y}+E u_{y}+F u_{x}+P u_{x}^{2}+Q u_{y}^{2}+G,
$$

where the coefficients $A, B, C, D, E, F, P, Q, G$ and $R \neq 0$ are functions of the independent variables $t, x, y$ and the dependent variable $u=u(t, x, y)$. We have established conditions determining the selfadjointness and the conservation laws for important Ricci flow equation, the modified Ricci flow 
equation and the nonlinear heat equation 2D, [1]. So the present paper is a natural generalization of that.

Firstly we investigate the nonlinear self-adjointness of the general evolution equation (1.1) which is the most important point of applying the Ibragimov's method. Secondly we establish the conservation laws for the nonlinear self-adjoint (modified) hyperbolic geometric flow equation.

This paper is organized as follows. In Section 2 we present the Ibragimov's method, the important concept of nonlinear self-adjointness and conservation laws for a scalar equation of second order. In Section 3 we state the main result in this work. In Section 4 we establish the conservation laws for (modified) hyperbolic geometric flow equation. Finally, the proof of the main theorem is presented in Appendix.

\section{The Ibragimov's Method}

In this section we develop the Ibragimov's theory for a single second order partial differential equation in the independent variables $t, x$ and $y$, dependent variable $u=u(t, x, y)$. For a general equation the basic literature recommended are the papers [4-11].

We define a differential function as $[5,6,8,11]$.

Definition 2.1. Let $t, x, y$ be the independent variables, $u=u(t, x, y)$ the dependent variable, $u_{t}, u_{x}$, $u_{y}, u_{x x}, u_{x y}, u_{y y}$, etc., its partial derivatives. A function $\mathscr{F}$ in the variable $t, x, y, u, u_{t}, u_{x}, u_{y}, u_{x x}, u_{x y}$, $u_{y y}$, etc., is called a differential function if it is locally analytic, i.e., if it admit locally a Taylor series expansion.

The concept of formal Lagrangian is introduced according to $[5,10,11]$.

Definition 2.2. Let $\mathscr{F}$ be a differential function in the variable $t, x, y, u, u_{t}, u_{x}, u_{y}, u_{x x}, u_{x y}, u_{y y}$, etc., and $v=v(t, x, y)$ is the new dependent variable, known as the adjoint variable or nonlocal variable [5], the formal Lagrangian function for the $\mathscr{F}$ is the differential function in the variable $t$, $x, y, u, v, u_{t}, u_{x}, u_{y}, u_{x x}, u_{x y}, u_{y y}$, etc., defined by

$$
\mathscr{L}:=v \mathscr{F} .
$$

Definition 2.3. Let $\mathscr{F}$ be a differential function and the differential equation

$$
\mathscr{F}\left(t, x, y, u, u_{t}, u_{x}, u_{y}, u_{x x}, u_{x y}, u_{y y}, \cdots\right)=0,
$$

denoted by $\mathscr{F}[u]=0$, we define the adjoint differential function to $\mathscr{F}$ by

$$
\mathscr{F}^{*}:=\frac{\delta \mathscr{L}}{\delta u},
$$

and the adjoint differential equation by

$$
\mathscr{F}^{*}\left(t, x, y, u, v, u_{t}, v_{t}, u_{x}, v_{x}, u_{y}, v_{y}, u_{x x}, v_{x x}, u_{x y}, v_{x y}, u_{y y}, v_{y y}, \cdots\right)=0,
$$

denoted by $\mathscr{F}^{*}[u, v]=0$, where

$$
\frac{\delta}{\delta u}=\frac{\partial}{\partial u}-\mathscr{D}_{t} \frac{\partial}{\partial u_{t}}-\mathscr{D}_{x} \frac{\partial}{\partial u_{x}}-\mathscr{D}_{y} \frac{\partial}{\partial u_{y}}+\mathscr{D}_{x} \mathscr{D}_{y} \frac{\partial}{\partial u_{x y}}+\mathscr{D}_{t}^{2} \frac{\partial}{\partial u_{t t}}+\mathscr{D}_{x}^{2} \frac{\partial}{\partial u_{x x}}+\mathscr{D}_{y}^{2} \frac{\partial}{\partial u_{y y}}-\cdots
$$


is the Euler-Lagrange operator, and

$$
\begin{aligned}
& \mathscr{D}_{t}=\frac{\partial}{\partial t}+u_{t} \frac{\partial}{\partial u}+v_{t} \frac{\partial}{\partial v}+u_{t t} \frac{\partial}{\partial u_{t}}+v_{t t} \frac{\partial}{\partial v_{t}}+u_{t x} \frac{\partial}{\partial u_{x}}+v_{t x} \frac{\partial}{\partial v_{x}}+u_{t y} \frac{\partial}{\partial u_{y}}+\cdots, \\
& \mathscr{D}_{x}=\frac{\partial}{\partial x}+u_{x} \frac{\partial}{\partial u}+v_{x} \frac{\partial}{\partial v}+u_{t x} \frac{\partial}{\partial u_{t}}+v_{t x} \frac{\partial}{\partial v_{t}}+u_{x x} \frac{\partial}{\partial u_{x}}+v_{x x} \frac{\partial}{\partial v_{x}}+u_{x y} \frac{\partial}{\partial u_{y}}+\cdots, \\
& \mathscr{D}_{y}=\frac{\partial}{\partial y}+u_{y} \frac{\partial}{\partial u}+v_{y} \frac{\partial}{\partial v}+u_{t y} \frac{\partial}{\partial u_{t}}+v_{t y} \frac{\partial}{\partial v_{t}}+u_{x y} \frac{\partial}{\partial u_{x}}+v_{x y} \frac{\partial}{\partial v_{x}}+u_{y y} \frac{\partial}{\partial u_{y}}+\cdots,
\end{aligned}
$$

are the total derivative operator with respect to $t, x$ and $y$, respectively.

Definition 2.4. The differential equation (2.2) is said to be nonlinearly self-adjoint if there exists a function

$$
v=\varphi(t, x, y, u) \neq 0
$$

such that it satisfy

$$
\left.\mathscr{F}^{*}\right|_{\nu=\varphi(t, x, y, u)}=\lambda \mathscr{F}
$$

for some undetermined coefficient $\lambda=\lambda(t, x, y, u, \cdots)$. If $v=\varphi(u)$ in (2.6) and (2.7), the equation (2.1) is called quasi self-adjoint. If $v=u$, we say that the equation (2.1) is strictly self-adjoint.

Definition 2.5. A conservation law for equation $\mathscr{F}[u]=0$ is the total divergence

$$
\operatorname{Div}(C)=\mathscr{D}_{t} C^{1}+\mathscr{D}_{x} C^{2}+\mathscr{D}_{y} C^{3},
$$

for some vectorial differential function $C[u]=\left(C^{1}, C^{2}, C^{3}\right)$, so-called conserved vector, such that the divergence vanishes for all solutions $u=f(t, x, y)$ for equation $\mathscr{F}[u]=0$. A conservation law is said to be nonlocal conservation law if the conserved vector depends on the solutions $v$ of the adjoint equation (2.4).

Now we state the New Conservation Theorem (or Theorem on nonlocal conservation laws, [8]) for the differential equation (2.4).

Theorem 2.1. Every Lie point and Lie-Bäcklund symmetry

$$
X=\tau(t, x, y, u, \cdots) \frac{\partial}{\partial t}+\xi(t, x, y, u, \cdots) \frac{\partial}{\partial x}+\eta(t, x, y, u, \cdots) \frac{\partial}{\partial y}+\phi(t, x, y, u, \cdots) \frac{\partial}{\partial u},
$$

as well as nonlocal symmetry, of the differential equation $\mathscr{F}[u]=0$, provides a nonlocal conservation law

$$
\operatorname{Div}(C)=0 \quad \text { for } \quad \mathscr{F}[u]=0 \quad \text { and } \quad \mathscr{F}^{*}[u, v]=0 .
$$

Let $W=\phi-\tau u_{t}-\xi u_{x}-\eta u_{y}$ be the Lie characteristic, then the components of the conserved vector, $C[u]=\left(C^{1}, C^{2}, C^{3}\right)$, are given by

$$
\begin{aligned}
& C^{1}-\tau \mathscr{L}=W\left(\frac{\partial \mathscr{L}}{\partial u_{t}}-\mathscr{D}_{t} \frac{\partial \mathscr{L}}{\partial u_{t t}}\right)+\mathscr{D}_{t} W \frac{\partial \mathscr{L}}{\partial u_{t t}}, \\
& C^{2}-\xi \mathscr{L}=W\left(\frac{\partial \mathscr{L}}{\partial u_{x}}-\mathscr{D}_{x} \frac{\partial \mathscr{L}}{\partial u_{x x}}-\mathscr{D}_{y} \frac{\partial \mathscr{L}}{\partial u_{x y}}\right)+\mathscr{D}_{x} W \frac{\partial \mathscr{L}}{\partial u_{x x}}+\mathscr{D}_{y} W \frac{\partial \mathscr{L}}{\partial u_{x y}} \\
& C^{3}-\eta \mathscr{L}=W\left(\frac{\partial \mathscr{L}}{\partial u_{y}}-\mathscr{D}_{x} \frac{\partial \mathscr{L}}{\partial u_{y x}}-\mathscr{D}_{y} \frac{\partial \mathscr{L}}{\partial u_{y y}}\right)+\mathscr{D}_{x} W \frac{\partial \mathscr{L}}{\partial u_{y x}}+\mathscr{D}_{y} W \frac{\partial \mathscr{L}}{\partial u_{y y}} .
\end{aligned}
$$


We note that $\mathscr{L}$ vanishes on the solutions of the equation (2.4), then the term $\tau \mathscr{L}, \xi \mathscr{L}$, and $\eta \mathscr{L}$ may be omitted in the conserved vector.

\section{The Main 2D Generalized Quasilinear Equation}

In this section we apply to the equation (1.1) the Ibragimov's method. For this purpose we write this equation in the form (2.1), where

$$
\mathscr{F}:=u_{t t}-A u_{x y}-B u_{x} u_{y}-C u_{x x}-D u_{y y}-E u_{y}-F u_{x}-P u_{x}^{2}-Q u_{y}^{2}-G-H u_{t}-I u_{t}^{2} .
$$

Then the corresponding formal Lagrangian (2.2) is given by

$$
\mathscr{L}=v\left(u_{t t}-A u_{x y}-B u_{x} u_{y}-C u_{x x}-D u_{y y}-E u_{y}-F u_{x}-P u_{x}^{2}-Q u_{y}^{2}-G-H u_{t}-I u_{t}^{2}\right)
$$

and the Euler-Lagrange operator (2.5) assumes the following form:

$$
\frac{\delta}{\delta u}=\frac{\partial}{\partial u}-\mathscr{D}_{t} \frac{\partial}{\partial u_{t}}-\mathscr{D}_{x} \frac{\partial}{\partial u_{x}}-\mathscr{D}_{y} \frac{\partial}{\partial u_{y}}+\mathscr{D}_{t}^{2} \frac{\partial}{\partial u_{t t}}+\mathscr{D}_{x}^{2} \frac{\partial}{\partial u_{x x}}+\mathscr{D}_{x} \mathscr{D}_{y} \frac{\partial}{\partial u_{x y}}+\mathscr{D}_{y}^{2} \frac{\partial}{\partial u_{y y}}+\ldots
$$

Now we apply the Euler-Lagrange operator (3.3) to $\mathscr{L}$ determined by (3.2) and after some work we obtain that the adjoint equation (2.4) to (1.1) (see Proposition 5.2 in Appendix) reads:

$$
\begin{aligned}
\mathscr{F}^{*} & =v_{t t}-A v_{x y}-C v_{x x}-D v_{y y}+\left(H+2 I u_{t}\right) v_{t} \\
& +\left[\left(B-A_{u}\right) u_{y}+2\left(P-C_{u}\right) u_{x}+F-A_{y}-2 C_{x}\right] v_{x} \\
& +\left[\left(B-A_{u}\right) u_{x}+2\left(Q-D_{u}\right) u_{y}+E-A_{x}-2 D_{y}\right] v_{y} \\
& +\left[2 I u_{t t}+2\left(B-A_{u}\right) u_{x y}+\left(B_{u}-A_{u u}\right) u_{x} u_{y}+2\left(P-C_{u}\right) u_{x x}\right. \\
& +2\left(Q-D_{u}\right) u_{y y}+\left(B_{x}-A_{x u}+2\left(Q_{y}-D_{y u}\right)\right) u_{y} \\
& +\left(B_{y}-A_{y u}+2\left(P_{x}-C_{x u}\right)\right) u_{x}+\left(P_{u}-C_{u u}\right) u_{x}^{2} \\
& +\left(Q_{u}-D_{u u}\right) u_{y}^{2}-A_{x y}-C_{x x}-D_{y y} \\
& \left.+E_{y}+F_{x}+H_{t}-G_{u}+2 I_{t} u_{t}+I_{u} u_{t}^{2}\right] v=0 .
\end{aligned}
$$

Therefore we get the main result in the present paper, which can be stated as follows.

Theorem 3.1. The eq. (1.1) is nonlinearly self-adjoint if and only if there exists a two time differentiable function $\varphi=\varphi(t, x, y, u) \neq 0$ such that its coefficients satisfies the following relations:

$$
\varphi_{u}+\varphi I=0,
$$

if at least the function $A_{1}, B_{1}, C_{1}, D_{1}, E_{1}, F_{1}$, defined below, is nonzero,

$$
\begin{gathered}
\varphi_{t u}+(\varphi I)_{t}+H\left(\varphi_{u}+\varphi I\right)=0, \\
\left((\varphi A)_{u}-\varphi B\right)_{y}+2\left((\varphi C)_{u}-\varphi P\right)_{x}-2 F\left(\varphi_{u}+\varphi I\right)=0, \\
\left((\varphi A)_{u}-\varphi B\right)_{x}+2\left((\varphi D)_{u}-\varphi Q\right)_{y}-2 E\left(\varphi_{u}+\varphi I\right)=0, \\
\varphi_{t t}-(\varphi A)_{x y}-(\varphi C)_{x x}-(\varphi D)_{y y}+(\varphi E)_{y}+(\varphi F)_{x}+(\varphi H)_{t}+\varphi_{u} G= \\
\varphi\left(G_{u}-2 G I\right),
\end{gathered}
$$


where $A, B, C, D, E, F, P, Q, G, H$ and I are function of $t, x, y, u$, and the functions $A_{1}, B_{1}, C_{1}, D_{1}$, $E_{1}, F_{1}$ are given by

$$
\begin{gathered}
A_{1}:=A_{u}, B_{1}:=D C_{u}-C D_{u}, C_{1}:=C_{u}, \\
D_{1}:=D_{u}, E_{1}:=A C_{u}-C A_{u}, F_{1}:=A D_{u}-D A_{u} .
\end{gathered}
$$

Further, the coefficient functions satisfy the following condictions:

$$
\begin{gathered}
A_{1}=A I+B, \\
B_{1}=D P-C Q, \\
C_{1}=C I+P, \\
D_{1}=D I+Q, \\
E_{1}=A P-C B, \\
F_{1}=A Q-D B .
\end{gathered}
$$

The quasi-self-adjointness and the strict self-adjointness of the studied equations are direct consequence of Theorem 3.1. Here we will apply the Theorem 3.1 to modified hyperbolic geometric flow equation, in particular, to hyperbolic geometric flow equation.

\section{The modified hyperbolic geometric flow equation}

In this section we apply the Theorem 3.1 to the equation

$$
u_{t t}=e^{\lambda u}\left(u_{x x}+u_{y y}\right)+\lambda u_{t}^{2},
$$

where $\lambda$ is a nonzero constant, and establish the corresponding conservation laws.

This equation is a natural generalization of the hyperbolic geometric flow equation, where $\lambda=$ -1 , and was suggested by professors Yuri Bozhkov and Igor Freire in [2].

The eq. (4.1) is of type (1.1) with

$$
C=D=e^{\lambda u}, \quad I=\lambda, \quad A=B=E=F=G=P=Q=H=0 .
$$

A first corollary of the Theorem 3.1 is the following:

Corollary 4.1. The equation

$$
u_{t t}=f(u)\left(u_{x x}+u_{y y}\right)+\lambda u_{t}^{2},
$$

where $f=f(u)$ and $\lambda$ do not vanish, is nonlinearly self-adjoint if and only if $f(u)=a e^{\lambda u}$ where a is nonzero constant. 
Kênio A. A. Silva

Proof. According the Theorem 3.1 we have

$$
\begin{aligned}
& A_{1}:=R A_{u}-A R_{u}=0, \\
& B_{1}:=D C_{u}-C D_{u}=0, \\
& C_{1}:=R C_{u}-C R_{u}=f^{\prime}(u), \\
& D_{1}:=R D_{u}-D R_{u}=C_{1}, \\
& E_{1}:=A C_{u}-C A_{u}=0, \\
& F_{1}:=A D_{u}-D A_{u}=0 .
\end{aligned}
$$

Further, the coefficient functions must satisfy the following relations:

$$
\begin{gathered}
A I+R B=0=A_{1}, \quad D P-C Q=0=B_{1}, \quad C I+R P=\lambda f(u)=C_{1}, \\
A P-C B=0=E_{1}, \quad A Q-D B=0=F_{1} .
\end{gathered}
$$

So we have $f^{\prime}(u)=\lambda f(u)$, i.e., $f(u)=a e^{\lambda u}$ where $a$ is a constant. As $C_{1} \neq 0$ from eq. (3.5) we obtain

$$
\varphi_{u}=-\lambda \varphi, \quad \text { i.e., } \quad \varphi=\varphi^{1} e^{-\lambda u},
$$

where $\varphi^{1}=\varphi^{1}(t, x, y)$ is any nonvanishing function.

The eqs. $(3.6,3.7,3.8)$ are satisfied trivially by $\varphi$. From eq. (3.9) we have

$$
0=\varphi_{t t}-\varphi_{x x} f(u)-\varphi_{y y} f(u)=\varphi_{t t}^{1} e^{-\lambda u}-a\left(\varphi_{x x}^{1}+\varphi_{y y}^{1}\right),
$$

this is,

$$
0=\varphi_{t t}^{1}=\varphi_{x x}^{1}+\varphi_{y y}^{1}
$$

Thus, $\varphi=(\alpha t+\beta) e^{-\lambda u}$ where $\alpha=\alpha(x, y)$ and $\beta=\beta(x, y)$ are any harmonic functions both nonvanishing.

Therefore, the eq. $u_{t t}=f(u)\left(u_{x x}+u_{y y}\right)+\lambda u_{t}^{2}$ is nonlinearly self-adjoint if and only if $f(u)=$ $a e^{\lambda u}$ where $a$ is nonzero constant.

Then we calculate some conservation laws for eq. (4.1). Its Lie symmetry are computed in Proposition 5.3 of Appendix, in terms of two functions satisfying the Cauchy-Riemann equations and the general infinitesimal generator of symmetries is given by

$$
X=\left(c_{1}+c_{4} t\right) \frac{\partial}{\partial t}+\xi(x, y) \frac{\partial}{\partial x}+\eta(x, y) \frac{\partial}{\partial y}+\frac{2}{\lambda}\left(\xi_{x}-c_{4}\right) \frac{\partial}{\partial u}
$$

where $c_{1}, c_{4}$ are arbitrary constants, $\xi_{x}=\eta_{y}$ and $\eta_{x}=-\xi_{y}$. 
We consider the following sub-algebra with infinitesimal generators of symmetries given by:

$$
\begin{gathered}
V_{1}=\frac{\partial}{\partial t}, \quad V_{2}=\frac{\partial}{\partial x}, \quad V_{3}=\frac{\partial}{\partial y}, V_{4}=t \frac{\partial}{\partial t}-\frac{2}{\lambda} \frac{\partial}{\partial u}, \\
V_{5}=x \frac{\partial}{\partial x}+y \frac{\partial}{\partial y}+\frac{2}{\lambda} \frac{\partial}{\partial u}, V_{6}=y \frac{\partial}{\partial x}-x \frac{\partial}{\partial y} .
\end{gathered}
$$

Let be the general generator

$$
V=\left(c_{1}+c_{4} t\right) \frac{\partial}{\partial t}+\left(c_{2}+c_{5} x+c_{6} y\right) \frac{\partial}{\partial x}+\left(c_{3}-c_{6} x+c_{5} y\right) \frac{\partial}{\partial y}+\frac{2}{\lambda}\left(c_{5}-c_{4}\right) \frac{\partial}{\partial u},
$$

where $c_{1}, c_{2}, c_{3}, c_{4}, c_{5}$ and $c_{6}$ are arbitrary constants.

Consider the adjoint variable $v=(\alpha t+\beta) e^{-\lambda u}$, where $\alpha=\alpha(x, y)$ and $\beta=\beta(x, y)$ are any harmonic functions both nonvanishing, the formal Lagrangian is given by

$$
\mathscr{L}=(\alpha t+\beta) e^{-\lambda u}\left(u_{t t}-e^{\lambda u}\left(u_{x x}+u_{y y}\right)-\lambda u_{t}^{2}\right),
$$

that is,

$$
\mathscr{L}=(\alpha t+\beta)\left(e^{-\lambda u}\left(u_{t t}-\lambda u_{t}^{2}\right)-u_{x x}-u_{y y}\right) .
$$

Now we apply to the conserved vectors given by New Conservation Theorem (Theorem 2.1), $C=\left(C^{1}, C^{2}, C^{3}\right)$, the simplifying operation described on pp. 50-51 of [11], and we obtain the following components:

$$
\begin{aligned}
C^{1} & =\left[\frac{2}{\lambda}\left(c_{4} \alpha+\alpha_{x}\left(c_{2}+c_{5} x+c_{6} y\right)\right)-\left[\alpha_{x}\left(c_{2}+c_{5} x+c_{6} y\right)+\alpha_{y}\left(c_{3}-c_{6} x+c_{5} y\right)\right] u\right] e^{-\lambda u} \\
& +e^{-\lambda u}\left\{-2 \alpha c_{5} u+\left[\left(\alpha_{x} t+\beta_{x}\right)\left(c_{2}+c_{5} x+c_{6} y\right)+\left(\alpha_{y} t+\beta_{y}\right)\left(c_{3}-c_{6} x+c_{5} y\right)\right.\right. \\
& \left.+\alpha\left(c_{1}+c_{4} t\right)+(\alpha t+\beta) c_{4}\right] u_{t}+(\lambda u-2) \alpha\left(c_{2}+c_{5} x+c_{6} y\right) u_{x} \\
& \left.+\lambda u \alpha\left(c_{3}-c_{6} x+c_{5} y\right) u_{y}\right\}, \\
C^{2} & =\left(\frac{2}{\lambda}-u\right) a\left(\alpha_{y y} t+\beta_{y y}\right)\left(c_{2}+c_{5} x+c_{6} y\right)+a\left(\alpha_{x} t+\beta_{x}\right)\left(\frac{2}{\lambda}\left(c_{5}-c_{4}\right)+\left(c_{5}+c_{4}\right) u\right) \\
& +a\left[\left(c_{1}+c_{4} t\right) \alpha_{x}+\left(\alpha_{x y} t+\beta_{x y}\right)\left(c_{3}-c_{6} x+c_{5} y\right)-\left(\alpha_{y} t+\beta_{y}\right) c_{6}\right] u \\
& -a\left[\left(\alpha_{x} t+\beta_{x}\right)\left(c_{2}+c_{5} x+c_{6} y\right)+\left(\alpha_{y} t+\beta_{y}\right)\left(c_{3}-c_{6} x+c_{5} y\right)+\alpha\left(c_{1}+c_{4} t\right)\right. \\
& \left.+(\alpha t+\beta) c_{4}\right] u_{x}-(\lambda u-2) \alpha\left(c_{2}+c_{5} x+c_{6} y\right) e^{-\lambda u} u_{t}, \\
C^{3} & =\left(u-\frac{2}{\lambda}\right) a\left(\alpha_{x y} t+\beta_{x y}\right)\left(c_{2}+c_{5} x+c_{6} y\right)+a\left(\alpha_{y} t+\beta_{y}\right)\left(-\frac{2}{\lambda} c_{4}+\left(c_{5}+c_{4}\right) u\right) \\
& +\left(u+\frac{2}{\lambda}\right) a\left(\alpha_{x} t+\beta_{x}\right) c_{6}+a\left[\left(c_{1}+c_{4} t\right) \alpha_{y}-\left(\alpha_{x x} t+\beta_{x x}\right)\left(c_{3}-c_{6} x+c_{5} y\right)\right] u \\
& -a\left[\left(\alpha_{x} t+\beta_{x}\right)\left(c_{2}+c_{5} x+c_{6} y\right)+\left(\alpha_{y} t+\beta_{y}\right)\left(c_{3}-c_{6} x+c_{5} y\right)+\alpha\left(c_{1}+c_{4} t\right)\right. \\
& \left.+(\alpha t+\beta) c_{4}\right] u_{y}-\lambda u \alpha\left(c_{3}-c_{6} x+c_{5} y\right) e^{-\lambda u} u_{t} .
\end{aligned}
$$

Therefore we prove the first corollary of Theorem 2.1: 
Corollary 4.2. Let $V$ be an infinitesimal generator of Lie symmetry for the eq.(4.1), give by

$$
V=\left(c_{1}+c_{4} t\right) \frac{\partial}{\partial t}+\left(c_{2}+c_{5} x+c_{6} y\right) \frac{\partial}{\partial x}+\left(c_{3}-c_{6} x+c_{5} y\right) \frac{\partial}{\partial y}+\frac{2}{\lambda}\left(c_{5}-c_{4}\right) \frac{\partial}{\partial u},
$$

where $c_{1}, c_{2}, c_{3}, c_{4}, c_{5}, c_{6}$ are arbitrary constants, $\lambda$ is a nonzero constant, and let $\alpha=\alpha(x, y)$ and $\beta=\beta(x, y)$ be harmonic functions both nonvanishing, then the corresponding nonlocal conserved vectors are the following:

- For generator $V_{1}$, the components of the conserved vector $C=\left(C^{1}, C^{2}, C^{3}\right)$, are given by

$$
C^{1}=e^{-\lambda u} \alpha u_{t}, C^{2}=\alpha_{x} u-\alpha u_{x}, C^{3}=\alpha_{y} u-\alpha u_{y} .
$$

- For generator $V_{2}$, the components of the conserved vector $C=\left(C^{1}, C^{2}, C^{3}\right)$, are given by

$$
\begin{aligned}
& C^{1}=\left[\frac{2}{\lambda} \alpha_{x}-\alpha_{x} u+\left(\alpha_{x} t+\beta_{x}\right) u_{t}+(\lambda u-2) \alpha u_{x}\right] e^{-\lambda u}, \\
& C^{2}=\left(\frac{2}{\lambda}-u\right)\left(\alpha_{y y} t+\beta_{y y}\right)-\left(\alpha_{x} t+\beta_{x}\right) u_{x}-(\lambda u-2) \alpha e^{-\lambda u} u_{t}, \\
& C^{3}=\left(u-\frac{2}{\lambda}\right)\left(\alpha_{x y} t+\beta_{x y}\right)-\left(\alpha_{x} t+\beta_{x}\right) u_{y} .
\end{aligned}
$$

- For generator $V_{3}$, the components of the conserved vector $C=\left(C^{1}, C^{2}, C^{3}\right)$, are given by

$$
\begin{aligned}
& C^{1}=\left[-\alpha_{y} u+\left(\alpha_{y} t+\beta_{y}\right) u_{t}+\lambda u \alpha u_{y}\right] e^{-\lambda u}, \\
& C^{2}=\left(\alpha_{x y} t+\beta_{x y}\right) u-\left(\alpha_{y} t+\beta_{y}\right) u_{x}, \\
& C^{3}=-\left(\alpha_{x x} t+\beta_{x x}\right) u-\left(\alpha_{y} t+\beta_{y}\right) u_{y}-\lambda u \alpha e^{-\lambda u} u_{t} .
\end{aligned}
$$

- For generator $V_{4}$, the components of the conserved vector $C=\left(C^{1}, C^{2}, C^{3}\right)$, are given by

$$
\begin{aligned}
C^{1} & =\left[\frac{2}{\lambda} \alpha u+(2 \alpha t+\beta) u_{t}\right] e^{-\lambda u}, \\
C^{2} & =\left(u-\frac{2}{\lambda}\right)\left(\alpha_{x} t+\beta_{x}\right)+t \alpha_{x} u-(2 \alpha t+\beta) u_{x} \\
C^{3} & =\left(u-\frac{2}{\lambda}\right)\left(\alpha_{y} t+\beta_{y}\right)+t \alpha_{y} u-(2 \alpha t+\beta) u_{y} .
\end{aligned}
$$

- For generator $V_{5}$, the components of the conserved vector $C=\left(C^{1}, C^{2}, C^{3}\right)$, are given by

$$
\begin{aligned}
C^{1} & =\left[\frac{2}{\lambda} \alpha_{x} x-\left(\alpha_{x} x+\alpha_{y} y+2 \alpha\right) u+\left[\left(\alpha_{x} t+\beta_{x}\right) x+\left(\alpha_{y} t+\beta_{y}\right) y\right] u_{t}\right. \\
& \left.+(\lambda u-2) \alpha x u_{x}+\lambda u \alpha y u_{y}\right] e^{-\lambda u}, \\
C^{2} & =\left(\frac{2}{\lambda}-u\right)\left(\alpha_{y y} t+\beta_{y y}\right) x+\left(\frac{2}{\lambda}+u\right)\left(\alpha_{x} t+\beta_{x}\right) \\
& +\left(\alpha_{x y} t+\beta_{x y}\right) y u-\left[\left(\alpha_{x} t+\beta_{x}\right) x+\left(\alpha_{y} t+\beta_{y}\right) y\right] u_{x}-(\lambda u-2) \alpha x e^{-\lambda u} u_{t}, \\
C^{3} & =\left(u-\frac{2}{\lambda}\right)\left(\alpha_{x y} t+\beta_{x y}\right) x+\left[\alpha_{y} t+\beta_{y}-\left(\alpha_{x x} t+\beta_{x x}\right) y\right] u \\
& -\left[\left(\alpha_{x} t+\beta_{x}\right) x+\left(\alpha_{y} t+\beta_{y}\right) y\right] u_{y}-\lambda u \alpha y e^{-\lambda u} u_{t} .
\end{aligned}
$$


- For generator $V_{6}$, the components of the conserved vector $C=\left(C^{1}, C^{2}, C^{3}\right)$, are given by

$$
\begin{aligned}
C^{1} & =\left[\frac{2}{\lambda} \alpha_{x} y-\left(\alpha_{x} y-\alpha_{y} x\right) u+\left[\left(\alpha_{x} t+\beta_{x}\right) y-\left(\alpha_{y} t+\beta_{y}\right) x\right] u_{t}\right. \\
& \left.+(\lambda u-2) \alpha y u_{x}-\lambda u \alpha x u_{y}\right] e^{-\lambda u}, \\
C^{2} & =\left(\frac{2}{\lambda}-u\right)\left(\alpha_{y y} t+\beta_{y y}\right) y-\left[\left(\alpha_{x y} t+\beta_{x y}\right) x+\alpha_{y} t+\beta_{y}\right] u \\
& -\left[\left(\alpha_{x} t+\beta_{x}\right) y-\left(\alpha_{y} t+\beta_{y}\right) x\right] u_{x}-(\lambda u-2) \alpha y e^{-\lambda u} u_{t}, \\
C^{3} & =\left(u-\frac{2}{\lambda}\right)\left(\alpha_{x y} t+\beta_{x y}\right) y+\left(\frac{2}{\lambda}+u\right)\left(\alpha_{x} t+\beta_{x}\right) \\
& +\left(\alpha_{x x} t+\beta_{x x}\right) x u-\left[\left(\alpha_{x} t+\beta_{x}\right) y-\left(\alpha_{y} t+\beta_{y}\right) x\right] u_{y}+\lambda u \alpha x e^{-\lambda u} u_{t} .
\end{aligned}
$$

We note that local conservation laws can be obtained taking $\alpha=0$ and $\beta$ a nonzero constant. A second corollary of the Theorem 3.1 is the follows:

Corollary 4.3. The hyperbolic geometric flow equation, $u_{t t}=e^{-u}\left(u_{x x}+u_{y y}\right)-u_{t}^{2}$, is quasi-selfadjoint.

Proof. Follow from Corollary 4.1 with $f(u)=e^{-u}, \lambda=-1, \alpha=0$ and $\beta$ a nonzero constant.

A second corollary of the Theorem 2.1 is the follows:

Corollary 4.4. Let $X$ be the general infinitesimal generator of Lie symmetry for the hyperbolic geometric flow equation, give by

$$
X=\left(c_{1}+c_{4} t\right) \frac{\partial}{\partial t}+\xi(x, y) \frac{\partial}{\partial x}+\eta(x, y) \frac{\partial}{\partial y}-2\left(\xi_{x}-c_{4}\right) \frac{\partial}{\partial u},
$$

where $c_{1}, c_{4}$ are arbitrary constants, $\xi_{x}=\eta_{y}, \eta_{x}=-\xi_{y}$, then the corresponding nonlocal conserved vector $C=\left(C^{1}, C^{2}, C^{3}\right)$ is give by

$$
C^{1}=e^{u} u_{t}, \quad C^{2}=-u_{x}, \quad C^{3}=-u_{y} .
$$

Proof. The general infinitesimal generator $X$ is obtained in Proposition 5.3. Consider the adjoint variable $v=\beta e^{u}$, where $\beta$ is a nonzero constant, then we obtain the following components for the conserved vectors:

$$
\begin{aligned}
C^{1} & =\beta e^{u}\left\{\left[2\left(c_{4}-\xi_{x}\right)-\left(c_{1}+c_{4} t\right) u_{t}-\xi u_{x}-\eta u_{y}\right] u_{t}\right. \\
& \left.-c_{4} u_{t}-\left(c_{1}+c_{4} t\right)\left(e^{-u}\left(u_{x x}+u_{y y}\right)-u_{t}^{2}\right)-\xi u_{t x}-\eta u_{t y}\right\}, \\
C^{2} & =\beta\left[2 \xi_{x x}+\left(c_{1}+c_{4} t\right) u_{x t}+\xi_{x} u_{x}+\eta_{x} u_{y}+\xi u_{x x}+\eta u_{x y}\right], \\
C^{3} & =\beta\left[2 \xi_{y x}+\left(c_{1}+c_{4} t\right) u_{y t}+\xi_{y} u_{x}+\eta_{y} u_{y}+\xi u_{y x}+\eta u_{y y}\right] .
\end{aligned}
$$

Now apply to the conserved vectors $C=\left(C^{1}, C^{2}, C^{3}\right)$ the simplifying operation described on pp. $50-51$ of [11] for obtain the following conserved vector, which is generated by

$$
X=t \frac{\partial}{\partial t}+2 \frac{\partial}{\partial u}
$$

and its components are given by

$$
C^{1}=e^{u} u_{t}, \quad C^{2}=-u_{x}, \quad C^{3}=-u_{y} .
$$


We note that this corollary can be obtained from Corollary 4.2 considering $\lambda=-1$, and the adjoint variable $v=\beta e^{u}$, with $\alpha=0$ and $\beta$ is a nonzero constant, we obtain a only non-trivial conserved vector which is generated by $V_{4}$.

\section{Appendix}

Proposition 5.1. The adjoint equation to $\mathscr{F}=0$, where $\mathscr{F}$ is determined by (3.1), is given by (3.4).

Proof. Recall the formal Lagrangian is given by

$$
\begin{aligned}
\mathscr{L} & =v\left(u_{t t}-A u_{x y}-B u_{x} u_{y}-C u_{x x}-D u_{y y}-E u_{y}-F u_{x}-P u_{x}^{2}-Q u_{y}^{2}-G\right. \\
& \left.-H u_{t}-I u_{t}^{2}\right) .
\end{aligned}
$$

See (3.2). Then the corresponding partial derivatives of $\mathscr{L}$ are given by

$$
\begin{aligned}
& \frac{\partial \mathscr{L}}{\partial u}= v\left(-A_{u} u_{x y}-B_{u} u_{x} u_{y}-C_{u} u_{x x}-D_{u} u_{y y}-E_{u} u_{y}-F_{u} u_{x}-P_{u} u_{x}^{2}-Q_{u} u_{y}^{2}\right. \\
&\left.-G_{u}-H_{u} u_{t}-I_{u} u_{t}^{2}\right), \\
& \frac{\partial \mathscr{L}}{\partial u_{t}}=-v\left(H+2 I u_{t}\right), \frac{\partial \mathscr{L}}{\partial u_{x}}=-v\left(B u_{y}+2 P u_{x}+F\right), \frac{\partial \mathscr{L}}{\partial u_{y}}=-v\left(2 Q u_{y}+B u_{x}+E\right), \\
& \frac{\partial \mathscr{L}}{\partial u_{t t}}=v, \quad \frac{\partial \mathscr{L}}{\partial u_{x x}}=-v C, \quad \frac{\partial \mathscr{L}}{\partial u_{y y}}=-v D, \quad \frac{\partial \mathscr{L}}{\partial u_{x y}}=-v A .
\end{aligned}
$$


The total derivatives involved in the Euler operator are given by

$$
\begin{aligned}
-\mathscr{D}_{t} \frac{\partial \mathscr{L}}{\partial u_{t}}= & \left(2 I u_{t}+H\right) v_{t}+\left(2 I u_{t t}+\left(H_{u}+2 I_{t}\right) u_{t}+2 I_{u} u_{t}^{2}+H_{t}\right) v, \\
-\mathscr{D}_{x} \frac{\partial \mathscr{L}}{\partial u_{x}}= & \left(B u_{y}+2 P u_{x}+F\right) v_{x}+\left(B u_{x y}+B_{u} u_{x} u_{y}+2 P u_{x x}+B_{x} u_{y}+\left(F_{u}+2 P_{x}\right) u_{x}\right. \\
& \left.+2 P_{u} u_{x}^{2}+F_{x}\right) v \\
-\mathscr{D}_{y} \frac{\partial \mathscr{L}}{\partial u_{y}}= & \left(2 Q u_{y}+B u_{x}+E\right) v_{y}+\left(B u_{x y}+B_{u} u_{x} u_{y}+2 Q u_{y y}+\left(E_{u}+2 Q_{y}\right) u_{y}+B_{y} u_{x}\right. \\
& \left.+2 Q_{u} u_{y}^{2}+E_{y}\right) v \\
\mathscr{D}_{t} \frac{\partial \mathscr{L}}{\partial u_{t t}}= & v_{t}, \quad \mathscr{D}_{t}^{2} \frac{\partial \mathscr{L}}{\partial u_{t t}}=v_{t t}, \\
\mathscr{D}_{x} \frac{\partial \mathscr{L}}{\partial u_{x y}}= & -A v_{x}-\left(A_{x}+A_{u} u_{x}\right) v, \\
\mathscr{D}_{y} \mathscr{D}_{x} \frac{\partial \mathscr{L}}{\partial u_{x y}}= & -A v_{x y}-\left(A_{x}+A_{u} u_{x}\right) v_{y}-\left(A_{y}+A_{u} u_{y}\right) v_{x}-\left(A_{u} u_{x y}+A_{u u} u_{x} u_{y}+A_{x u} u_{y}\right. \\
& \left.+A_{y u} u_{x}+A_{x y}\right) v, \\
\mathscr{D}_{x} \frac{\partial \mathscr{L}}{\partial u_{x x}}= & -C v_{x}-\left(C_{x}+C_{u} u_{x}\right) v, \\
\mathscr{D}_{x}^{2} \frac{\partial \mathscr{L}}{\partial u_{x x}}= & -C v_{x x}-2\left(C_{x}+C_{u} u_{x}\right) v_{x}-\left(C_{u} u_{x x}+C_{u u} u_{x}^{2}+2 C_{x u} u_{x}+C_{x x}\right) v, \\
\mathscr{D}_{y} \frac{\partial \mathscr{L}}{\partial u_{y y}}= & -D v_{y}-\left(D_{y}+D_{u} u_{y}\right) v, \\
\mathscr{D}_{y}^{2} \frac{\partial \mathscr{L}}{\partial u_{y y}}= & -D v_{y y}-2\left(D_{y}+D_{u} u_{y}\right) v_{y}-\left(D_{u} u_{y y}+2 D_{y u} u_{y}+D_{u u} u_{y}^{2}+D_{y y}\right) v,
\end{aligned}
$$

Substituting these formulae in $\mathscr{F}^{*}=\frac{\delta \mathscr{L}}{\delta u}$, we obtain the adjoint equation (3.4).

Proposition 5.2. The self-adjointness determining equations of the equation (1.1) are given by:

$$
\begin{aligned}
u_{t t}: & \varphi_{u}+2 I \varphi=\lambda, \\
u_{x y}: & -A \varphi_{u}+2\left(B-A_{u}\right) \varphi=-\lambda A, \\
u_{x} u_{y}: & -A \varphi_{u u}+2\left(B-A_{u}\right) \varphi_{u}+\left(B_{u}-A_{u u}\right) \varphi=-\lambda B, \\
u_{x x}: & -C \varphi_{u}+2\left(P-C_{u}\right) \varphi=-\lambda C, \\
u_{y y}: & -D \varphi_{u}+2\left(Q-D_{u}\right) \varphi=-\lambda D, \\
u_{y}: & -A \varphi_{x u}-2 D \varphi_{y u}+\left(B-A_{u}\right) \varphi_{x}+2\left(Q-D_{u}\right) \varphi_{y} \\
& +\left(E-A_{x}-2 D_{y}\right) \varphi_{u}+\left(B_{x}-A_{x u}+2\left(Q_{y}-D_{y u}\right)\right) \varphi=-\lambda E, \\
u_{x}: & -A \varphi_{y u}-2 C \varphi_{x u}+\left(B-A_{u}\right) \varphi_{y}+2\left(P-C_{u}\right) \varphi_{x} \\
& +\left(F-A_{y}-2 C_{x}\right) \varphi_{u}+\left(B_{y}-A_{y u}+2\left(P_{x}-C_{x u}\right)\right) \varphi=-\lambda F, \\
u_{t}: & 2 \varphi_{t u}+2 I \varphi_{t}+H \varphi_{u}+2 I_{t} \varphi=-\lambda H, \\
u_{x}^{2}: & -C \varphi_{u u}+2\left(P-C_{u}\right) \varphi_{u}+\left(P_{u}-C_{u u}\right) \varphi=-\lambda P, \\
u_{y}^{2}: & -D \varphi_{u u}+2\left(Q-D_{u}\right) \varphi_{u}+\left(Q_{u}-D_{u u}\right) \varphi=-\lambda Q,
\end{aligned}
$$


Kênio A. A. Silva

$$
\begin{aligned}
u_{t}^{2}: & \varphi_{u u}+2 I \varphi_{u}+I_{u} \varphi=-\lambda I, \\
1: & \varphi_{t t}-A \varphi_{x y}-C \varphi_{x x}-D \varphi_{y y}+\left(E-A_{x}-2 D_{y}\right) \varphi_{y} \\
& +\left(F-A_{y}-2 C_{x}\right) \varphi_{x}+H \varphi_{t} \\
& -\left(A_{x y}+C_{x x}+D_{y y}-E_{y}-F_{x}-H_{t}+G_{u}\right) \varphi=-\lambda G,
\end{aligned}
$$

for some differenciable function $\varphi=\varphi(t, x, y, u) \neq 0$ and undetermined coefficient $\lambda=$ $\lambda(t, x, y, u)$.

Proof. Substituting $v=\varphi(t, x, y, u)$ and its partial derivatives $v_{t}, v_{x}, v_{y}, v_{x x}, v_{x y}$, and $v_{y y}$ into $\mathscr{F}^{*}$ given by Proposition 5.1 we have

$$
\begin{aligned}
\left.\mathscr{F}^{*}\right|_{v=\varphi(t, x, y, u)} & =\varphi_{t t}+2 \varphi_{t u} u_{t}+\varphi_{u u} u_{t}^{2}+\varphi_{u} u_{t t} \\
& -A\left(\varphi_{x y}+\varphi_{x u} u_{y}+\varphi_{y u} u_{x}+\varphi_{u u} u_{x} u_{y}+\varphi_{u} u_{x y}\right) \\
& -C\left(\varphi_{x x}+2 \varphi_{x u} u_{x}+\varphi_{u u} u_{x}^{2}+\varphi_{u} u_{x x}\right) \\
& -D\left(\varphi_{y y}+2 \varphi_{y u} u_{y}+\varphi_{u u} u_{y}^{2}+\varphi_{u} u_{y y}\right) \\
& +\left(\left(B-A_{u}\right) u_{y}+2\left(P-C_{u}\right) u_{x}+F-A_{y}-2 C_{x}\right)\left(\varphi_{x}+\varphi_{u} u_{x}\right) \\
& +\left(\left(B-A_{u}\right) u_{x}+2\left(Q-D_{u}\right) u_{y}+E-A_{x}-2 D_{y}\right)\left(\varphi_{y}+\varphi_{u} u_{y}\right) \\
& +\left(H+2 I u_{t}\right)\left(\varphi_{t}+\varphi_{u} u_{t}\right) \\
& +\left[2 I u_{t t}+2\left(B-A_{u}\right) u_{x y}+\left(B_{u}-A_{u u}\right) u_{x} u_{y}+2\left(P-C_{u}\right) u_{x x}\right. \\
& +2\left(Q-D_{u}\right) u_{y y}+\left(B_{x}-A_{x u}+2\left(Q_{y}-D_{y u}\right)\right) u_{y} \\
& +\left(B_{y}-A_{y u}+2\left(P_{x}-C_{x u}\right)\right) u_{x}+\left(P_{u}-C_{u u}\right) u_{x}^{2} \\
& +\left(Q_{u}-D_{u u}\right) u_{y}^{2}-A_{x y}-C_{x x}-D_{y y} \\
& \left.+E_{y}+F_{x}+H_{t}-G_{u}+2 I_{t} u_{t}+I_{u} u_{t}^{2}\right] \varphi
\end{aligned}
$$

That is,

$$
\begin{aligned}
\left.\mathscr{F}^{*}\right|_{v=\varphi(t, x, y, u)} & =\left(\varphi_{u}+2 I \varphi\right) u_{t t}-\left(A \varphi_{u}-2\left(B-A_{u}\right) \varphi\right) u_{x y} \\
& -\left(A \varphi_{u u}-2\left(B-A_{u}\right) \varphi_{u}-\left(B_{u}-A_{u u}\right) \varphi\right) u_{x} u_{y} \\
& -\left(C \varphi_{u}-2\left(P-C_{u}\right) \varphi\right) u_{x x}+\left(D \varphi_{u}-2\left(Q-D_{u}\right) \varphi\right) u_{y y} \\
& -\left[A \varphi_{x u}+2 D \varphi_{y u}-\left(B-A_{u}\right) \varphi_{x}-2\left(Q-D_{u}\right) \varphi_{y}\right. \\
& \left.-\left(E-A_{x}-2 D_{y}\right) \varphi_{u}-\left(B_{x}-A_{x u}+2\left(Q_{y}-D_{y u}\right)\right) \varphi\right] u_{y} \\
& -\left[A \varphi_{y u}+2 C \varphi_{x u}-\left(B-A_{u}\right) \varphi_{y}-2\left(P-C_{u}\right) \varphi_{x}\right. \\
& \left.-\left(F-A_{y}-2 C_{x}\right) \varphi_{u}-\left(B_{y}-A_{y u}+2\left(P_{x}-C_{x u}\right)\right) \varphi\right] u_{x} \\
& +\left(2 \varphi_{t u}+2 I \varphi_{t}+H \varphi_{u}+2 I_{t} \varphi\right) u_{t} \\
& -\left[C \varphi_{u u}-2\left(P-C_{u}\right) \varphi_{u}-\left(P_{u}-C_{u u}\right) \varphi\right] u_{x}^{2} \\
& -\left[D \varphi_{u u}-2\left(Q-D_{u}\right) \varphi_{u}-\left(Q_{u}-D_{u u}\right) \varphi\right] u_{y}^{2} \\
& +\left(\varphi_{u u}+2 I \varphi_{u}+I_{u} \varphi\right) u_{t}^{2} \\
& +\varphi_{t t}-A \varphi_{x y}-C \varphi_{x x}-D \varphi_{y y}+\left(E-A_{x}-2 D_{y}\right) \varphi_{y} \\
& +\left(F-A_{y}-2 C_{x}\right) \varphi_{x}+H \varphi_{t} \\
& -\left(A_{x y}+C_{x x}+D_{y y}-E_{y}-F_{x}-H_{t}+G_{u}\right) \varphi .
\end{aligned}
$$


Now we equalize the coefficients of the monomials $u_{t t}, u_{x y}, u_{x} u_{y}, u_{x x}, u_{y y}, u_{y}, u_{x}, u_{x}^{2}, u_{y}^{2}, u_{t}, u_{t}^{2}$ and 1 in both sides the eq. (2.7) for we obtain the self-adjointness determining equations.

Proof. of Theorem 3.1. The eq. (3.10) is obtained adding to eq. (5.2) the eq. (5.1) multiplied by $A$.

The eq. (3.11) is obtained adding to eq. (5.4) multiplied by $D$ the eq. (5.5) multiplied by $-C$.

The eq. (3.12) is obtained adding to eq. (5.4) the eq. (5.1) multiplied by $C$.

The eq. (3.13) is obtained adding to eq. (5.5) the eq. (5.1) multiplied by $D$.

The eq. (3.14) is obtained adding to eq. (5.2) multiplied by $C$ the eq. (5.4) multiplied by $-A$.

The eq. (3.15) is obtained adding to eq. (5.2) multiplied by $D$ the eq. (5.5) multiplied by $-A$.

If $A_{1} \neq 0$ the eq. (3.5) is obtained adding to eq. (5.3) the eq. (5.11) multiplied by $A$, after we substitute the eqs. $(3.10,5.1)$ in the resultant equation obtaining the follows: $A_{u}\left(\varphi_{u}+I \varphi\right)=0$, this is, $\varphi_{u}+I \varphi=0$.

If $B_{1} \neq 0$ the eq. (3.5) is obtained adding to eq. (5.9) multiplied by $D$ the eq. (5.10) multiplied by $-C$, after we substitute the eqs. $(3.11,3.12,3.13,5.1)$ in the resultant equation obtaining the follows: $(D P-C Q)\left(\varphi_{u}+I \varphi\right)=0$, this is, $\varphi_{u}+I \varphi=0$.

If $C_{1} \neq 0$ the eq. (3.5) is obtained adding to eq. (5.9) the eq. (5.11) multiplied by $C$, after we substitute the eqs. $(3.12,5.1)$ in the resultant equation obtaining the follows: $C_{u}\left(\varphi_{u}+I \varphi\right)=0$, this is, $\varphi_{u}+I \varphi=0$.

If $D_{1} \neq 0$ the eq. (3.5) is obtained adding to eq. (5.10) the eq. (5.11) multiplied by $D$, after we substitute the eqs. $(3.13,5.1)$ in the resultant equation obtaining the follows: $D_{u}\left(\varphi_{u}+I \varphi\right)=0$, ou seja, $\varphi_{u}+I \varphi=0$.

If $E_{1} \neq 0$ the eq. (3.5) is obtained adding to eq. (5.3) multiplied by $C$ the eq. (5.9) multiplied by $-A$, after we substitute the eqs. $(3.10,3.12,3.14,5.1)$ in the resultant equation obtaining the follows: $(A P-C B)\left(\varphi_{u}+I \varphi\right)=0$, this is, $\varphi_{u}+I \varphi=0$.

If $F_{1} \neq 0$ the eq. (3.5) is obtained adding to eq. (5.3) multiplied by $D$ the eq. (5.10) multiplied by $-A$, after we substitute the eqs. $(3.10,3.13,3.15,5.1)$ in the resultant equation obtainig the follows: $(A Q-D B)\left(\varphi_{u}+I \varphi\right)=0$, this is, $\varphi_{u}+I \varphi=0$.

The eq. (3.6) is obtained substituting the eq. (5.1) into (5.8).

The eq. (3.7) is obtained substituting the eq. (5.1) in (5.7).

The eq. (3.8) is obtained substituting the eq. (5.1) in (5.6).

Finally, the eq. (3.9) is obtained substituting the eq. (5.1) in (5.12).

Proposition 5.3. The Lie symmetries of the modified hyperbolic geometric flow equation (4.1) are generate by following infinitesimal generator:

$$
X=\left(c_{1}+c_{4} t\right) \frac{\partial}{\partial t}+\xi(x, y) \frac{\partial}{\partial x}+\eta(x, y) \frac{\partial}{\partial y}+\frac{2}{\lambda}\left(\xi_{x}-c_{4}\right) \frac{\partial}{\partial u}
$$

where $c_{1}, c_{4}$ are arbitrary constants, and the functions $\xi=\xi(x, y)$ and $\eta=\eta(x, y)$ satisfies the Cauchy-Riemann equations:

$$
\xi_{x}=\eta_{y}, \quad \eta_{x}=-\xi_{y} .
$$

Proof. We will follow the notation of [13], Theorem 2.36, pg. 110. Let be the infinitesimal generator

$$
X=\tau \frac{\partial}{\partial t}+\xi \frac{\partial}{\partial x}+\eta \frac{\partial}{\partial y}+\phi \frac{\partial}{\partial u}
$$


where $\tau=\tau(t, x, y, u), \xi=\xi(t, x, y, u), \eta=\eta(t, x, y, u)$ and $\phi=\phi(t, x, y, u)$, its infinitesimal generator of the second prolongation is

$$
\mathbf{p r}^{(2)} X=\tau \frac{\partial}{\partial t}+\xi \frac{\partial}{\partial x}+\eta \frac{\partial}{\partial y}+\phi \frac{\partial}{\partial u}+\phi^{t} \frac{\partial}{\partial u_{t}}+\phi^{t t} \frac{\partial}{\partial u_{t t}}+\phi^{x x} \frac{\partial}{\partial u_{x x}}+\phi^{y y} \frac{\partial}{\partial u_{y y}}+\cdots
$$

where

$$
\begin{aligned}
\phi^{t} & =\mathscr{D}_{t} \phi-\left(\mathscr{D}_{t} \tau\right) u_{t}-\left(\mathscr{D}_{t} \xi\right) u_{x}-\left(\mathscr{D}_{t} \eta\right) u_{y} \\
& =\phi_{t}+\left(\phi_{u}-\tau_{t}\right) u_{t}-\tau_{u} u_{t}^{2}-\xi_{t} u_{x}-\eta_{t} u_{y}-\xi_{u} u_{t} u_{x}-\eta_{u} u_{t} u_{y}, \\
\phi^{t t} & =\mathscr{D}_{t} \phi^{t}-\left(\mathscr{D}_{t} \tau\right) u_{t t}-\left(\mathscr{D}_{t} \xi\right) u_{t x}-\left(\mathscr{D}_{t} \eta\right) u_{t y} \\
& =\phi_{t t}+\left(2 \phi_{t u}-\tau_{t t}\right) u_{t}-\xi_{t t} u_{x}-\eta_{t t} u_{y}+\left(\phi_{u u}-2 \tau_{t u}\right) u_{t}^{2}-2 \xi_{t u} u_{t} u_{x}-2 \eta_{t u} u_{t} u_{y} \\
& -\tau_{u u} u_{t}^{3}-\xi_{u u} u_{t}^{2} u_{x}-\eta_{u u} u_{t}^{2} u_{y}+\left(\phi_{u}-2 \tau_{t}-3 \tau_{u} u_{t}-\xi_{u} u_{x}-\eta_{u} u_{y}\right) u_{t t} \\
& -2\left(\xi_{t}+\xi_{u} u_{t}\right) u_{t x}-2\left(\eta_{t}+\eta_{u} u_{t}\right) u_{t y}, \\
\phi^{x x} & =\phi_{x x}+\left(2 \phi_{x u}-\xi_{x x}\right) u_{x}-\tau_{x x} u_{t}-\eta_{x x} u_{y}+\left(\phi_{u u}-2 \xi_{x u}\right) u_{x}^{2}-2 \tau_{x u} u_{t} u_{x}-2 \eta_{x u} u_{x} u_{y} \\
& -\xi_{u u} u_{x}^{3}-\tau_{u u} u_{x}^{2} u_{t}-\eta_{u u} u_{x}^{2} u_{y}+\left(\phi_{u}-2 \xi_{x}-3 \xi_{u} u_{x}-\tau_{u} u_{t}-\eta_{u} u_{y}\right) u_{x x} \\
& -2\left(\tau_{x}+\tau_{u} u_{x}\right) u_{t x}-2\left(\eta_{x}+\eta_{u} u_{x}\right) u_{x y} \\
\phi^{y y} & =\phi_{y y}+\left(2 \phi_{y u}-\eta_{y y}\right) u_{y}-\tau_{y y} u_{t}-\xi_{y y} u_{x}+\left(\phi_{u u}-2 \eta_{y u}\right) u_{y}^{2}-2 \tau_{y u} u_{t} u_{y}-2 \xi_{y u} u_{x} u_{y} \\
& -\eta_{u u} u_{y}^{3}-\tau_{u u} u_{y}^{2} u_{t}-\xi_{u u} u_{y}^{2} u_{x}+\left(\phi_{u}-2 \eta_{y}-3 \eta_{u} u_{y}-\tau_{u} u_{t}-\xi_{u} u_{x}\right) u_{y y} \\
& -2\left(\tau_{y}+\tau_{u} u_{y}\right) u_{t y}-2\left(\xi_{y}+\xi_{u} u_{y}\right) u_{x y} .
\end{aligned}
$$

Applying $\mathbf{p r}^{(2)} X$ to eq.(4.1) we find the infinitesimal criterion of invariance (Theorem 2.31, pg 104) must be satisfied whenever $u_{t t}-e^{\lambda u}\left(u_{x x}+u_{y y}\right)-\lambda u_{t}^{2}=0$, that is,

$$
\operatorname{pr}^{(2)} X\left(u_{t t}-e^{\lambda u}\left(u_{x x}+u_{y y}\right)-\lambda u_{t}^{2}\right)=0, \quad \text { whenever } \quad u_{t t}-e^{\lambda u}\left(u_{x x}+u_{y y}\right)-\lambda u_{t}^{2}=0 .
$$

Thus we must have

$$
\begin{aligned}
0 & =\left(\phi \frac{\partial}{\partial u}+\phi^{t} \frac{\partial}{\partial u_{t}}+\phi^{t t} \frac{\partial}{\partial u_{t t}}+\phi^{x x} \frac{\partial}{\partial u_{x x}}+\phi^{y y} \frac{\partial}{\partial u_{y y}}\right)\left(u_{t t}-e^{\lambda u}\left(u_{x x}+u_{y y}\right)-\lambda u_{t}^{2}\right) \\
& =-\phi \lambda e^{\lambda u}\left(u_{x x}+u_{y y}\right)-\phi^{t} 2 \lambda u_{t}+\phi^{t t}-\left(\phi^{x x}+\phi^{y y}\right) e^{\lambda u}
\end{aligned}
$$

Now we substitute the eq.(4.1) into $\phi^{t t}$ and the rewritten as

$$
\phi^{t t}:=\tilde{\phi}^{t t}+\left(\phi_{u}-2 \tau_{t}-3 \tau_{u} u_{t}-\xi_{u} u_{x}-\eta_{u} u_{y}\right)\left(e^{\lambda u}\left(u_{x x}+u_{y y}\right)+\lambda u_{t}^{2}\right)
$$

where $\tilde{\phi}_{u_{t t}}^{t t}=0$.

The coefficients of the quadratic monomials $u_{t} u_{x x}, u_{x} u_{x x}$ and $u_{y} u_{y y}$ in $(5.13,5.14)$ are given by

$$
\begin{aligned}
& u_{t} u_{x x}:-3 \tau_{u} \lambda e^{\lambda u}+\tau_{u} \lambda e^{\lambda u}=0 \\
& u_{x} u_{x x}:-\xi_{u} \lambda e^{\lambda u}+3 \xi_{u} \lambda e^{\lambda u}=0, \\
& u_{y} u_{y y}: \quad-\eta_{u} \lambda e^{\lambda u}+3 \eta_{u} \lambda e^{\lambda u}=0
\end{aligned}
$$


From $\lambda \neq 0$ follows that $\tau=\tau(t, x, y), \xi=\xi(t, x, y)$ and $\eta=\eta(t, x, y)$. Then we have

$$
\begin{aligned}
\phi^{t} & =\phi_{t}+\left(\phi_{u}-\tau_{t}\right) u_{t}-\xi_{t} u_{x}-\eta_{t} u_{y}, \\
\phi^{t t} & =\phi_{t t}+\left(2 \phi_{t u}-\tau_{t t}\right) u_{t}-\xi_{t t} u_{x}-\eta_{t t} u_{y}+\phi_{u u} u_{t}^{2}-2 \xi_{t} u_{t x}-2 \eta_{t} u_{t y} \\
& +\left(\phi_{u}-2 \tau_{t}\right)\left(e^{\lambda u}\left(u_{x x}+u_{y y}\right)+\lambda u_{t}^{2}\right) \\
& =\phi_{t t}+\left(2 \phi_{t u}-\tau_{t t}\right) u_{t}-\xi_{t t} u_{x}-\eta_{t t} u_{y}+\left(\phi_{u u}+\lambda\left(\phi_{u}-2 \tau_{t}\right)\right) u_{t}^{2}-2 \xi_{t} u_{t x}-2 \eta_{t} u_{t y} \\
& +\left(\phi_{u}-2 \tau_{t}\right) e^{\lambda u}\left(u_{x x}+u_{y y}\right), \\
\phi^{x x} & =\phi_{x x}+\left(2 \phi_{x u}-\xi_{x x}\right) u_{x}-\tau_{x x} u_{t}-\eta_{x x} u_{y}+\phi_{u u} u_{x}^{2}+\left(\phi_{u}-2 \xi_{x}\right) u_{x x}-2 \tau_{x} u_{t x} \\
& -2 \eta_{x} u_{x y} \\
\phi^{y y} & =\phi_{y y}+\left(2 \phi_{y u}-\eta_{y y}\right) u_{y}-\tau_{y y} u_{t}-\xi_{y y} u_{x}+\phi_{u u} u_{y}^{2}+\left(\phi_{u}-2 \eta_{y}\right) u_{y y}-2 \tau_{y} u_{t y} \\
& -2 \xi_{y} u_{x y}, \\
\phi^{x x}+\phi^{y y} & =\Delta \phi-\Delta \tau u_{t}+\left(2 \phi_{x u}-\Delta \xi\right) u_{x}+\left(2 \phi_{y u}-\Delta \eta\right) u_{y}+\phi_{u u}\left(u_{x}^{2}+u_{y}^{2}\right) \\
& +\left(\phi_{u}-2 \xi_{x}\right) u_{x x}+\left(\phi_{u}-2 \eta_{y}\right) u_{y y}-2 \tau_{x} u_{t x}-2 \tau_{y} u_{t y}-2\left(\eta_{x}+\xi_{y}\right) u_{x y} .
\end{aligned}
$$

Substituting this formulae in (5.13) we obtain

$$
\begin{aligned}
0 & =-\phi \lambda e^{\lambda u}\left(u_{x x}+u_{y y}\right)-\left(\phi_{t}+\left(\phi_{u}-\tau_{t}\right) u_{t}-\xi_{t} u_{x}-\eta_{t} u_{y}\right) 2 \lambda u_{t}+\phi_{t t}+\left(2 \phi_{t u}-\tau_{t t}\right) u_{t} \\
& -\xi_{t t} u_{x}-\eta_{t t} u_{y}+\left(\phi_{u u}+\lambda\left(\phi_{u}-2 \tau_{t}\right)\right) u_{t}^{2}-2 \xi_{t} u_{t x}-2 \eta_{t} u_{t y} \\
& +\left(\phi_{u}-2 \tau_{t}\right) e^{\lambda u}\left(u_{x x}+u_{y y}\right)-\left[\Delta \phi-\Delta \tau u_{t}+\left(2 \phi_{x u}-\Delta \xi\right) u_{x}+\left(2 \phi_{y u}-\Delta \eta\right) u_{y}\right. \\
& +\phi_{u u}\left(u_{x}^{2}+u_{y}^{2}\right)+\left(\phi_{u}-2 \xi_{x}\right) u_{x x}+\left(\phi_{u}-2 \eta_{y}\right) u_{y y}-2 \tau_{x} u_{t x}-2 \tau_{y} u_{t y} \\
& \left.-2\left(\eta_{x}+\xi_{y}\right) u_{x y}\right] e^{\lambda u}
\end{aligned}
$$

From eq. (5.15) we find the determining equations for the symmetry to be the following:

$$
\begin{aligned}
& u_{t}^{2}: \quad-2 \lambda\left(\phi_{u}-\tau_{t}\right)+\phi_{u u}+\lambda\left(\phi_{u}-2 \tau_{t}\right)=0, \\
& u_{x}^{2}+u_{y}^{2}: \quad \phi_{u u}=0, \\
& u_{x x}: \quad-\phi \lambda e^{\lambda u}+\left(\phi_{u}-2 \tau_{t}\right) e^{\lambda u}-\left(\phi_{u}-2 \xi_{x}\right) e^{\lambda u}=0, \\
& u_{y y}: \quad-\phi \lambda e^{\lambda u}+\left(\phi_{u}-2 \tau_{t}\right) e^{\lambda u}-\left(\phi_{u}-2 \eta_{y}\right) e^{\lambda u}=0, \\
& u_{x y}: \quad 2\left(\eta_{x}+\xi_{y}\right) e^{\lambda u}=0, \\
& u_{t x}: \quad-2 \xi_{t}+2 \tau_{x} e^{\lambda u}=0, \\
& u_{t y}: \quad-2 \eta_{t}+2 \tau_{y} e^{\lambda u}=0, \\
& u_{t} u_{x}: \quad 2 \lambda \xi_{t}=0, \\
& u_{t} u_{y}: \quad 2 \lambda \eta_{t}=0, \\
& u_{t}: \quad-2 \lambda \phi_{t}+\left(2 \phi_{t u}-\tau_{t t}\right)+\Delta \tau e^{\lambda u}=0, \\
& u_{x}: \quad-\xi_{t t}-\left(2 \phi_{x u}-\Delta \xi\right) e^{\lambda u}=0 \text {, } \\
& u_{y}: \quad-\eta_{t t}-\left(2 \phi_{y u}-\Delta \eta\right) e^{\lambda u}=0 \text {, } \\
& \text { 1: } \quad \phi_{t t}-\Delta \phi e^{\lambda u}=0 .
\end{aligned}
$$

The solution of the determining equations is elementary. First substitute the second determining equation into first, so we have $\phi=\phi(t, x, y)$ since $\lambda \neq 0$.

The eighth and ninth equations shows that $\xi=\xi(x, y)$ and $\eta=\eta(x, y)$. Substituting this $\xi$ and $\eta$ in the sixth and seventh equations we find $\tau=\tau(t)$. 
Now we solve the third equation for $\phi$ and we obtain

$$
-\left(\phi \lambda+2 \tau_{t}-2 \xi_{x}\right) e^{\lambda u}=0, \quad \text { that is, } \quad \phi=\frac{2}{\lambda}\left(\xi_{x}-\tau_{t}\right) .
$$

Subtracting to third the fourth equation, we have $\xi_{x}-\eta_{y}=0$. This and from fifth equation follow that the functions $\xi=\xi(x, y)$ and $\eta=\eta(x, y)$ satisfy the Cauchy-Riemann equations.

Substituting $\tau$ and $\phi$ in tenth equation, we obtain $\tau_{t t}=4 \tau_{t t}$, that is, $\tau=c_{1}+c_{4} t$, where $c_{1}$ and $c_{4}$ are arbitrary constants.

Thus, $\tau=c_{1}+c_{4} t$, the functions $\xi=\xi(x, y)$ and $\eta=\eta(x, y)$ satisfy the Cauchy-Riemann equations, and finally $\phi=\frac{2}{\lambda}\left(\xi_{x}-c_{4}\right)$.

\section{Acknowledgements}

Would like to thank the Prof. Dr. Yuri Bozhkov for his useful suggestions. This work was supported by CNPq, National Council for Scientific and Technological Development - Brazil.

\section{References}

[1] Y. Bozhkov, K. A. A. Silva, Nonlinear self-adjointness of a 2D generalized second order evolution equation, Nonlinear Analysis 75 (2012), 5069-5078.

[2] Y. Bozhkov, I. L. Freire, Private Communication, February 2012.

[3] X. Chao, Symmetries and geometric flows, arXiv:1001.1394 v1 [math.GT], 09 Jan 2010.

[4] M. L. Gandarias, Weak self-adjoint differential equations, J. Phys. A: Math. Theor. 44 (2011) 262001, $6 \mathrm{pp}$.

[5] N. H. Ibragimov, The answer to the question put to me by L.V. Ovsyannikov 33 years ago, Archives of ALGA 3 (2006), 55-60.

[6] — Integrating factors, adjoint equations and Lagrangians, J. Math. Anal. Appl. 318 (2006), 742757.

[7] — Quasi-self-adjoint differential equations, Archives of ALGA 4 (2007), 55-60.

[8] $\longrightarrow$ A new conservation theorem, J. Math. Anal. Appl. 333 (2007), 311-328.

[9] N. H. Ibragimov, R. S. Khamitova and A. Valenti, Self-adjointness of a generalized Camassa-Holm equation, Appl. Math. Comp., 218, (2011) 2579-2583.

[10] N. H. Ibragimov, Nonlinear self-adjointness and conservation laws, J. Phys. A: Math. Theor, 44, 432002, (2011), 8 pp..

[11] — Nonlinear self-adjointness in constructing conservation laws, Archives of ALGA 7/8 (20102011), 1-90.

[12] D. Kong, K. Liu, Wave character of metrics and hyperbolic geometric flow, J. Math. Phys. 48 (2007).

[13] P. J. Olver, Applications of Lie groups to differential equations, 2nd ed. GMT 107, Springer-Verlag New York, (1986).

[14] J. Wang, Symmetries and solutions of geometric flows, (2011). 